\title{
Neonatal Kolestaz ile Başvuran Konjenital Hipofiz Hormon Eksikliği Tanılı Yenidoğan Olgusu
}

A Newborn Presented with Cholestasis and Diagnosed with Congenital Pituitary Hormone Deficiency

\section{Elif OZALKAYA ${ }^{1}$, Arzu AKDAĞ ${ }^{2}$, Esra Deniz PAPATYA ${ }^{3}$, Sevilay TOPÇUOĞLU ${ }^{1}$}

1. Zeynep Kamil Kadın Doğum ve Çocuk Hastalıkları Eğitim ve Araştırma Hastanesi, Yenidoğan Yoğun Bakım Ünitesi, İstanbul

2. Bursa Yüksek İhtisas Eğitim ve Araştırma Hastanesi, Yenidoğan Yoğun Bakım Ünitesi, Bursa

3. Bursa Yüksek İhtisas Eğitim ve Araştırma Hastanesi, Çocuk Endokrinoloji, Bursa

\section{$\ddot{O Z Z T T}$}

Konjenital hipofiz hormon eksikliği neonatal kolestazın sık olmayan nedenidir. Yenidoğan döneminde klinik bulguları kolestaz ve hipoglisemidir. Sezeryan ile, 37 haftalı, $3700 \mathrm{gr}$ doğan kı bebekte postnatal birinci hafta hipoglisemi, 3. haftada kolestaz bulgularl gelişti. Çoklu hipofiz hormon eksikliği saptandl. Büyüme hormonu tedavisi ile kolestaz bulgularl düzeldi. Konjenital hipofiz hormon eksikliği, hipoglisemi ve kolestazı olan yenidoğanlarda ayırıcı tanıda akılda tutulmalıdir.

Anahtar Kelimeler: kolestaz; hipofiz; yenidoğan

\section{SUMMARY}

An infrequent reason of neonatal cholestasis is congenital pituitary hormone deficiency.

Clinical manifestations of cholestasis and hypoglycaemia developed in a girl baby born with caesarean section at gestational week 37, with a birth weigh of $3700 \mathrm{~g}$. Hypoglycemia symptoms developed at postnatal first and cholestasis at postnatal third week. Multiple pituitary hormone deficiency was identified. Cholestasis symptoms recovered with growth hormone therapy. Congenital pituitary hormone deficiency should be kept in mind in the newborns with hypoglycemia and cholestasis

Keywords: cholestasis; pituitary; newborn

\section{İletişim}

Sorumlu Yazar: Dr. Elif ÖZALKAYA

Adres: Zeynep Kamil Kadın Doğum ve Çoc. Hast. Eğt. ve Arş. Hast. Burhanettin Üstünel Cad. No:10 İstanbul, 34668, Türkiye Tel: +90 (533) 2162457

E-Posta: elifozalkay@gmail.com

Makale Geliș: 23.06.2016

Makale Kabul: 06.12.2016

DOI: http://dx.doi.org/10.16948/zktb.???????

\section{GíRis}

Neonatal kolestaz 1/2500 canlı doğumda bir görülmektedir (1). Sik olmayan nedenlerinden biri konjenital hipofiz hormon eksikliğidir. Konjenital hipofiz hormon eksikliğinde adrenokortikotropik hormon (ACTH), troid stimulan hormon (TSH), growth hormon $(\mathrm{GH})$, prolaktin, luteinizan hormon $(\mathrm{LH})$ /foliküler uyarıcı hormon $(\mathrm{FSH})$ ve antidiüretik hormon (ADH)'de hem izole hemde kombine eksiklikler meydana gelmektedir (2). Çoğul hipofiz hormon eksikliğine sekonder kolestazı olan yaklaşı 50 vaka bildirilmiştir $(3,4)$.

Kolestaza hangi hormon eksikliğinin neden olduğu açık değildir (5). Klinik olarak indirekt hiperbilurubinemi, sonrasinda direkt hiperbilurubinemi, kolestaz devam ederse karaciğer fonksiyon testlerinde yükselme meydana gelir (6). Hormon replesman tedavisi (HRT) ile kolestaz ve karaciğer fonksiyon testleri düzelmektedir (1). Biz bu olgu sunumunda neonatal kolestazın sık olmayan nedeni konjenital çoğul hipofiz hormon eksikliği olan bir yenidoğanı sunduk.

\section{OLGU}

Otuzyedi yaşındaki annenin üçüncü gebeliğinden üçüncü canlı doğum olarak 37. gestasyon haftasında ağırlığı 3900 gr (3,33 SDS), boyu $52 \mathrm{~cm}(1,22 \mathrm{SDS})$, baş çevresi $35 \mathrm{~cm}$ $(0,36$ SDS $)$ olarak, sezaryen $(\mathrm{C} / \mathrm{S})$ ile doğan k1z bebek postnatal 3. gününde hipoglisemi nedeni ile yenidoğan yoğun bakım servisine yatırıldı.

Hastanın fizik muayenesinde orta yüzde hipoplazi ve belirgin frontal çıkıklık mevcuttu. Anne ve babası birinci derece kuzen olan hastanın yedi ve beş yaşındaki iki erkek kardeşinde hastalık öyküsü yoktu. Hipoglisemi nedeni ile yatırılan hastaya $6 \mathrm{mg} / \mathrm{kg} / \mathrm{dk}$ glukoz infüzyonu başland. Total bilirubin $15 \mathrm{mg} / \mathrm{dl}(0-13 \mathrm{mg} / \mathrm{dl})$, indirekt bilirubin $14 \mathrm{mg} / \mathrm{dl}$ olan hastaya indirekt hiperbilirubinemisi nedeni ile 2 gün fototerapi verildi. Oral beslenen, hipoglisemisi ve hiperbilirubinemisi olmayan hasta taburcu edildi. 
22 günlük iken poliklinik kontrolünde total bilirubin $11,3 \mathrm{mg} / \mathrm{dl}(0-1 \mathrm{mg} / \mathrm{dl})$, direkt bilirubin 2,62 mg/dl (0- 0,3 mg/dl), AST:83 U/L (0$40 \mathrm{U} / \mathrm{L})$, ALT:33 U/L (0- $41 \mathrm{U} / \mathrm{L}), \mathrm{GGT}: 39 \mathrm{U} / \mathrm{L}$ (8- $61 \mathrm{U} / \mathrm{L})$ bulundu. Kolestaz nedeni ile ikinci kez yenidoğan yoğun bakım servisine yatırıldı. İki kez hipoglisemisi (venöz kan şekeri 37 ve $35 \mathrm{mg} / \mathrm{dl}$ ) olan hastanın hipoglisemi sirasında, insulin düzeyi $1,1 \mathrm{U} / \mathrm{ml}$, kortizol $1 \mu \mathrm{g} / \mathrm{dl}(2.8$ $23 \mu \mathrm{g} / \mathrm{dl})$ ve $\mathrm{GH} 1,65 \mathrm{ng} / \mathrm{ml}(7.91 \pm 5.57 \mathrm{ng} /$ $\mathrm{ml})$ düzeyleri saptand1. Tiroid fonksiyon testleri (TSH 6 mg/dl, serbest FT4: 0,53 mg/ dl ) santral hipotiroidi ile uyumlu olan hastanın prolaktin $(5,2 \mathrm{ng} / \mathrm{ml})(30-495 \mathrm{ng} / \mathrm{ml})$ düzeyi de yenidoğan dönemine göre düşük olarak değerlendirildi. Hastanın LH 0 IU/L $(0,50 \pm 0.25$ U/I), FSH $0.07 \mathrm{IU} / \mathrm{L}(7.07 \pm 5.92 \mathrm{IU} / \mathrm{I})$ bulundu (Tablo 1).

$\mathrm{Bu}$ bulgularla hastada çoğul hipofizer hormon eksikliği düşünüldü. Batın ultrasonu, ekokardiografi, Tandem Mass, idrar ve kan aminoasitleri, idrar organik asit analizi normal bulundu. Toxoplazma, rubella, sitomegalovirus ve herpes virüs serolojisi negatif idi. Hipofiz MR ' 1 normal bulundu. Hastaya 25. Gün hidrokortizon $10 \mathrm{mg} / \mathrm{m}^{2} /$ gün dozunda üç dozda başlandı. Bir hafta sonra hastaya 10 $\mu \mathrm{g} / \mathrm{kg} / \mathrm{gün}$ dozunda levotiroksin başlandı. Tablo 1: Konjenital hipofiz hormon eksikliği olan hastanın hormon profili.

\begin{tabular}{|c|c|c|c|c|c|c|c|}
\hline Postnatal & Kortizol & АСТН & TSH & PRL & $\mathrm{GH}$ & LH & FSH \\
\hline 22.gün & $\begin{array}{c}1 \\
(2.8-23) \\
\mu g / d l\end{array}$ & $\begin{array}{c}11,7 \\
(6-48) \\
\mathrm{pg} / \mathrm{ml}\end{array}$ & 1,89 & $\begin{array}{c}5,2 \\
(30-495) \\
\mathrm{ng} / \mathrm{ml}\end{array}$ & $\begin{array}{c}1,65 \\
(7.91 \pm 5.57) \\
\mathrm{ng} / \mathrm{mL}\end{array}$ & $\begin{array}{c}0.1 \\
(0,50 \pm 0.25) \\
U / I\end{array}$ & $\begin{array}{c}0,07 \\
(7.07 \pm 5.92) \\
I U / I\end{array}$ \\
\hline
\end{tabular}

Tablo 2: Hastanın hormon replasman tedavisi ile takipteki karaciğer fonksiyon testleri .

\begin{tabular}{|c|c|c|c|c|c|}
\hline & ALT & AST & GGT & T. Bİ & D. BíL \\
\hline 22. gün & $33 \mathrm{U} / \mathrm{L}$ & $83 \mathrm{U} / \mathrm{L}$ & $39 \mathrm{U} / \mathrm{L}$ & $11,3 \mathrm{mg} / \mathrm{dl}$ & $2,62 \mathrm{mg} / \mathrm{dl}$ \\
\hline \multicolumn{6}{|c|}{ 25. gün Levotroksin ve hidrokortizon } \\
\hline 48. gün & $30 \mathrm{U} / \mathrm{L}$ & $128 \mathrm{U} / \mathrm{L}$ & $39 \mathrm{U} / \mathrm{L}$ & $12,5 \mathrm{mg} / \mathrm{dl}$ & $8,5 \mathrm{mg} / \mathrm{dl}$ \\
\hline \multicolumn{6}{|l|}{ 50. gün GH } \\
\hline 120. gün & $20 \mathrm{U} / \mathrm{L}$ & $53 \mathrm{U} / \mathrm{L}$ & $17 \mathrm{U} / \mathrm{L}$ & $1 \mathrm{mg} / \mathrm{dl}$ & $0,3 \mathrm{mg} / \mathrm{dl}$ \\
\hline
\end{tabular}

Postnatal 48. gününde total bilirubin 12,5 $\mathrm{mg} / \mathrm{dl}$, direkt bilirubin $8,5 \mathrm{mg} / \mathrm{dl}$, aspartat aminotransferaz (AST) $128 \mathrm{U} / \mathrm{L}$, alanin aminotransferaz (ALT) $30 \mathrm{U} / \mathrm{L}$, gamaglutamil transpeptidaz (GGT) $39 \mathrm{U} / \mathrm{L}$ bulundu. L- tiroksin ve hidrokortizon almakta olan bebeğe postnatal 50. Gününde $\mathrm{GH} 0,35 \mathrm{mg} / \mathrm{kg} /$ hafta başlandi. $\mathrm{GH}$ başlandiktan sonra postnatal 120 günlük kolestaz bulguları düzeldi (total bilirubin 1 $\mathrm{mg} / \mathrm{dl}$, direkt bilirubin $0,3 \mathrm{mg} / \mathrm{dl}$, AST:53 U/L, ALT:20 U/L, GGT: 17 U/L) (Tablo 2, Şekil 1). Son kontrolünde 14 aylık olan hastanın boyu 70 cm (-2.16 SDS) ağırlığ $1,3 \mathrm{~kg}(-3.33 \mathrm{SDS})$ saptandi. Hastanın GH tedavisi $0,35 \mathrm{mg} / \mathrm{kg} / \mathrm{hafta}$, levotiroksin $8 \mu \mathrm{g} / \mathrm{kg} / \mathrm{gün}$, hidrokortizon $12 \mathrm{mg} /$ $\mathrm{m}^{2} /$ gün dozlarında devam etmektedir. Hastanın bir yaşında yapılan Denver gelişimsel değerlendirilmesi yaşıyla uyumlu saptanmıştır.

\section{TARTIŞMA}

Konjenital hipopituriazim çoğunlukla asemptomatiktir. Yenidoğan dönemindeki klinik bulguları letarji, kilo alımında azalma ve beslenme bozukluğudur. Y1llık insidansı yüzbinde 4,2' dir (7). Geciken tan1 ile tekrarlayan hipoglisemi ve kortizol eksikliği yenidoğan mortalitesini arttırmaktadır $(2,8)$. 


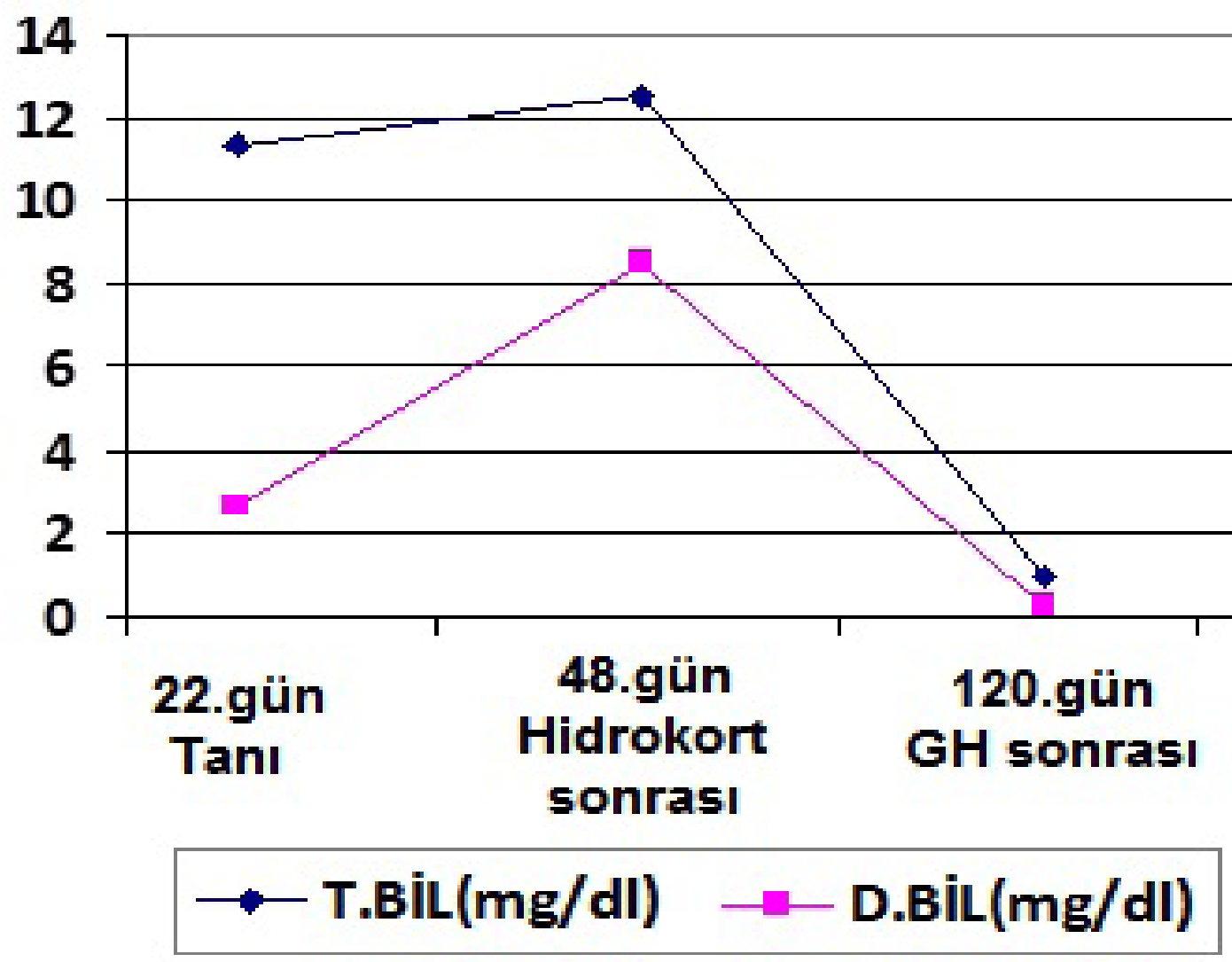

Şekil 1: Konjenital hipofiz hormon eksikliği olan hastanın, Hormon ReplasmanTedavisi ile kolestaz izlemi.

Biz bu olguyu hipoglisemi ve neonatal kolestazı olan yenidoğanlarda konjenital hipopituriazimin ayırıcı tanıda daha sık düşünülmesi için sunduk. Konjenital hipofiz hormon eksikliğinin hipoglisemi ve kolestaza neden olduğu ilk kez Blizzard ve Alberts tarafindan bildirilmiş (9), en geniş olgu serisi 7 yılda 16 hastayı içeren Braslavsky ve arkadaşları tarafindan sunulmuştur. Konjenital hipopituriazmi olan 16 hastanın 12'sinde kombine hipopituriazm saptanmıştır. Kolestaz başlangıç yaşı ortalama 18 gün, endokrinoloğa refere edilme ortalama 32 gün bulunmuștur. Konjuge bilurubin ortalama $4,85 \mathrm{mg} / \mathrm{dl}$ iken 10 hastanın transaminazlar1 2-10 katı artmış, GGT 9 hastada yükselmiş, 14'ünde spontan düzelen, 9' unda rekürren, 8 'inde ciddi semptomatik hipoglisemi öyküsü saptanmıştır. Kombine hormon eksikliği olan bebeklerin hipofiz Mr'inda anterior hipofizde hipoplazi, agenezi, ya da incelme, posterior hipofiz sinyalinde ektopi ya da yokluk saptanmiştır (1). Bizim merkezimizde 2 yıl içerisinde bir yenidoğana kombine hipopituriazim tanısı konulmuştur. Kolestaz başlangıç yaşı 22 gün, endokrinoloğa refere edilme 30 gündür. Konjuge bilurubin $8,5 \mathrm{mg} / \mathrm{dl}$, AST 3 kat artarken, ALT ve GGT hafif yükselmiş, rekürren hipoglisemisi olmuştur. Bizim hastanın Hipofiz MR'ında Braslavsky 'dan farklı olarak patoloji saptanmamıştır. Braslavsky ve arkadaşlarının çalışmasında kombine hipofiz hormon eksikliği olan tüm hastalara hidrokortizon ve levotiroksin başlanmış, ortalama 65 . günde kolestaz normale dönmüș. Ortalama 3,9 y1l izledikleri hastalarında normale dönmeyen karaciğer fonksiyon testi olmamıştır (1). Konjenital hipofiz hormon eksikliği olan infantlarda hormon replasman tedavisinin hidrokortizon, levotroksin ve büyüme hormonu etkilerini değerlendiren Binder ve arkadaşlarının çalışmasında 88 . Günde (10), Spray ve arkadaşlarının çalışmasinda (11) 42. Günde kolestaz bulgularında düzelme saptanmıştır. Bizim olgumuzda ise levotiroksin ve hidrokortizon tedavisi ile kolestaz ve karaciğer fonksiyon testleri düzelmemiştir. Braslavsky'dan farklı olarak GH başlandıktan 70 gün, postnatal 120. gününde kolestazı düzelmiştir. Konjenital hipofiz hormon eksikliğinde hangi hormon eksikliğinin kolestaza neden olduğu bilinmemektedir (5). Adrenalleri alınan hayvanlarda kortizol eksikliği ile safra akımının azaldığı gösterilmiştir. GH safra asit sekresyon ve biosentezini etkilemektedir (10). Bizim hastamızda GH'dan, Binder ve Spray'ın çalışmasında hidrokortizon, levotiroksin ve GH'dan sonra kolestazın düzelmesi nedeni ile konjenital hipofiz hormon eksikliğinde kolestazın düzelmesinde primer rol oynayan hormonun $\mathrm{GH}$ olduğu düşünülmüştür.

Sonuç olarak hipoglisemi ve neonatal kolestazı olan yenidoğanlarda konjenital hipofiz hormon eksikliği düşünülmelidir. Kolestazın remisyonu için kortizolden çok büyüme hormon replasman tedavisi geçiktirilmeden başlanmalidir. 


\section{KAYNAKLAR}

1. Braslavsky D, Keselman A, Galoppo M, Lezama C, Chiesa A, Galoppo C et.al. Neonatal cholestasis in congenital pituitary hormone deficiency and isolated hypocortisolism: characterization of liver dysfunction and follow-up. Arq Bras Endocrinol Metab. 2011;55:622-627

2. Choo-Kang LR, Sun CJ, Counts DR. Clinical case seminar. Cholestasis and hypoglycemia: manifestations of congenital anterior hypopituitarism. J Clin Endocrinol Metab. 1996;81:2786-2789.

3. Leblanc A, Odievre M, Hadchouel M, Gendrel D, Chaussain JL, Rappaport R. Neonatal cholestasis and hypoglycemia: possible role of cortisol deficiency. J Pediatr. 1981;99:577-580

4.De Salvo D, Pohl JF, Wilson DP, Byrant W, Easley D, Greene J. et.al. Cholestasis secondary to panhyopituitarism in an infant. J Natl Med Assoc 2008;100:342-344.

5. Bauman JW, Chang BS, HallBauman JW, Chang BS, Hall FR. The effects of adrenalectomy and hypophysectomy on bile flow in the rats. Acta Endocrinol. 1966;52:404.

6. Karnsakul W, Sawathiparnich P, Nimkarn S, Likitmaskul $S$, Santiprabhob J, Aanpreung P. Anterior pituitary hormone effects on hepatic functions in infants with congenital hypopituitarism. Annals of Hepathology. 2007;6:97-103.
7. Moyer V, Freese DK, Whitington PF, Olson AD, Brewer $F$, Colletti RB, et.al. North American Society for Pediatric Gastroenterology. Hepatology and Nutrition. Guideline for the evaluation of cholestasic jaundice in infants: recommendations of the North American society for pediatric gastroenterology, hepathology and nutrition. J Pediatr Gastroenterol Nutr. 2004;39:115128.

8. Ellaway CJ, Silink M, Cowell CT, Gaskin KJ, Kamath $K R$, Donaghue, et al. Cholestatic jaundice and congenital hypopituitarism. J Paediatr Child Health 1995 ;31:51-53.

9. Blizzard RM, Alberts M. Hypopituitarism, hypoadrenalism and hypogonadism in the newborn infant. J Pediatr. 1956;48:782-92.Paediatr Child Health. 1995;31:51-53.

10. Binder G, Martin DD, Kanther I, Schwarze CP, Ranke $M B$. The course of neonatal cholestasis in congenital combined pituitary hormone deficiency. J Pediatr Endocrinol Metab. 2007;20:695-701.

11. Spray CH, Mckiernan P, Waldron KE, Shaw N, Kirk $J$, Kelly DA. Investigation and outcome of neonatal hepatitis in infants with hypopituitarism. Acta Paediatr. 2000;89:951-954.
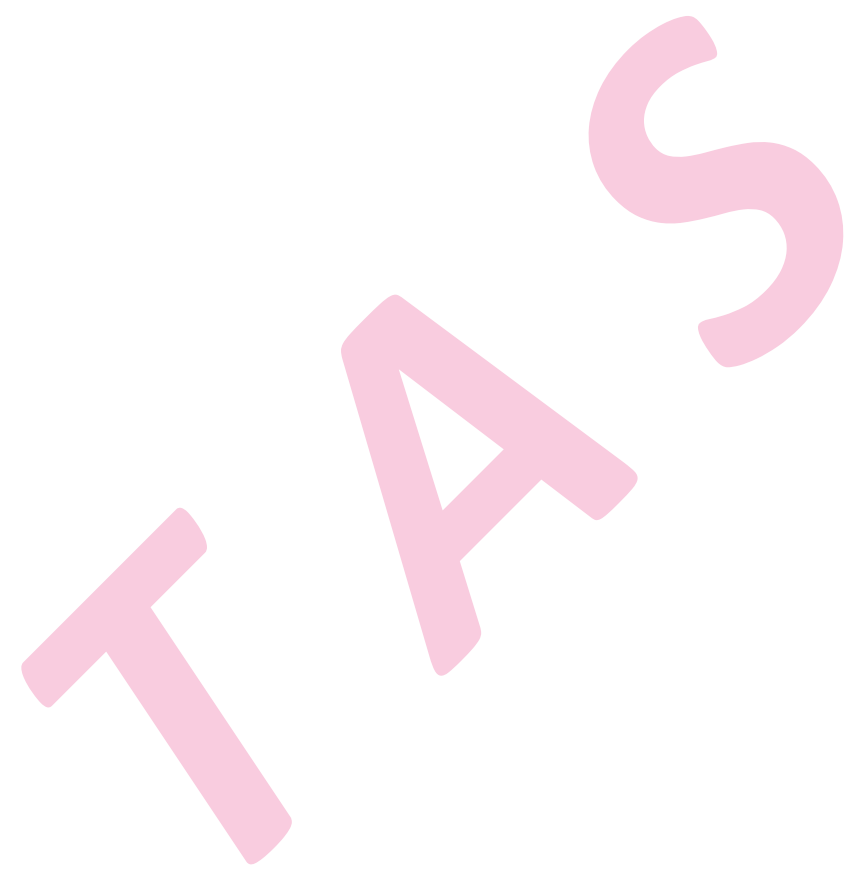